circulatory system was greater in mothers aged 20-34 years compared to mothers under 19 years of age (HR 5.64 95\% CI 1.65 to $19.27 ; \mathrm{p}=0.01$ ) and in babies with low birth weight (HR 3.09; 95\% CI 1.27 to 7.51). For digestive system anomalies mortality was associated with complications during pregnancy (HR 1.67; $95 \%$ CI 1.11 to 2.52; $\mathrm{p}=0.01$ ). For musculoskeletal system malformations mother's disease in pregnancy (HR 11.04; 95\% CI 1.31 to $9.30 ; p=0.03$ ) and complications during delivery (HR 18.98; $95 \%$ CI 2.39 to $15.04 ; p=0.00$ ) were associated with mortality.

Conclusions The risk factors identified highlight the importance of careful antenatal care.

\section{SP6-64 MORTALITY IN THE ELDERLY, DUE TO PROXIMAL FEMUR FRACTURE: 1-YEAR FOLLOW-UP STUDY}

doi:10.1136/jech.2011.142976q.35

${ }^{1} S$ Campos, ${ }^{1,2} \mathrm{M}$ F Pina, ${ }^{*}{ }^{1,4} \mathrm{~A}$ Trigo-Cabral. ${ }^{1}$ INEB-Instituto de Engenharia Biomédica, Porto, Portugal; ${ }^{2}$ ISPUP-Instituto de Saúde Pública da Universidade do Porto, Porto, Portugal; ${ }^{3}$ Serviço de Higiene e Epidemiologia-Faculdade de Medicina da Universidade do Porto, Porto, Portugal; ${ }^{4}$ Serviço de Ortopedia e Traumatologia do Hospital de São João, Porto, Portugal

Objectives Identify risk factors for mortality after hip fracture. Material and Methods Patients admitted in the orthopaedics service of the main hospital in Porto city, from 1 May 2008 to 30 April 2009, with a low-energy hip fracture were selected. During admission a questionnaire was applied and phone interviews to the patients or a close relative were done at 3, 6, 9 and 12 months after the fracture. From hospital registers, fracture type, surgery date, surgical treatment, co-morbilities and ASA score were obtained.

Results At admission, patients ( $\mathrm{n}=252,79 \%$ women, mean age of $80.3 \pm 9.5$ years and $76.3 \pm 11.3$ years $(p<0.05)$, women and men respectively) lived mainly with someone (67\%); $1 \%$ were confined to bed, $65 \%$ had difficulties in walking or doing daily activities and $34 \%$ had a life without restrictions. The most common co-morbidities were hypertension for women (52\% vs $38 \%$ ) and respiratory disease for men (36\% vs $11 \%$ ), $\mathrm{p}<0.05$. Death was $22 \%, 25 \%, 30 \%$ and $37 \%$ for men and $8 \%, 14 \%, 20 \%$ and $23 \%$ for women, respectively at 3,6 , 9 and 12 months of follow-up. Death was higher among institutionalised patients. Survival analysis using Kaplan-Meier curve and Cox regression analysis showed that the risk of dead increased $6 \%$ for each age-year older, $151 \%$ if patient was a man, $94 \%$ for ASA score III/IV and $7 \%$ for each day of delay to the surgery.

Conclusion Older age, male sex, ASA scores III/IV and delay to surgery are good predictors of mortality after a hip fracture.

\section{SP6-65 EMERGENCY MEDICAL SERVICE THROUGH COMMUNITY BASED VOLUNTEERS IN RURAL AREAS BANGLADESH}

doi:10.1136/jech.2011.142976q.36

${ }^{1} \mathrm{M}$ J Hossain, ${ }^{* 1,2} \mathrm{~A}$ K M Fazlur Rahman, ${ }^{1} \mathrm{~A}$ Rahman, ${ }^{1}$ S R Mashreky, ${ }^{1,3} \mathrm{~K}$ UI Baset, ${ }^{1} \mathrm{~A}$ Biswas, 'S Shafinaz. 'Centre for Injury Prevention and Research, Bangladesh, Dhaka, Bangladesh; ${ }^{2}$ Kerolinska Institute, Stockholm, Sweden; ${ }^{3}$ University of west England, Bristol, UK

Background Emergency medical service (EMS) cannot only lessen the severity of an illness but can also save lives. In this study we accessed the emergency medical services provided by trained community based volunteers in rural areas of Bangladesh. The objectives of this study were (1) To access the volunteer based emergency medical services and (2) To describe the types of emergency medical care services provided by the volunteers.

Methods In 2008, we trained community volunteers to deliver EMS within limited areas. A special emergency medical service manual was developed. We selected volunteers from the community and trained them for 3 days intensively. After training all volunteers were provided a first aid box fully equipped with medicine and materials. The first 1 to 3 months of data about medical services were collected from the volunteers.

Results A total of 136 volunteers worked and 1403 patients were provided emergency medical services. After burn injuries all patients were treated first with water, 72 patients mostly injured with cuts and falls were treated with clean water before starting other treatment. 1175 patient received an antiseptic wash among them $75 \%$ had a cut injury and $11.5 \%$ a fall injury. Among all patient $58 \%$ were provided with bandage and these patients mostly had a cut injury. The volunteers treated 8 patients with Cardiopulmonary Resuscitation. Analgesia was given mostly in cuts (60.2\%), falls (15.1\%) and RTI (3.5\%) patients and patients with fever $(5.3 \%)$. About 249 (18\%) of all patients were referred for further treatment. Conclusion Expanding emergency medical service through community volunteers will be very effective in reducing mortality, morbidity and progression of any complications after injury.

\section{SP6-66 THE RELATIONSHIP BETWEEN DENTAL DECAY AND CAREGIVER NEGLECT IN CHILDREN}

doi:10.1136/jech.2011.142976q.37

C Lourenço, ${ }^{*}$ M V Santrain, A V Meyer. Universidade de Fortaleza-UNIFOR, Fortaleza, Ceará/Nordeste, Brazil

Despite all the public health policies, dental decay continues to be one of the most prevalent diseases in children (Siqueira et al, 2009). Among the factors related to dental decay in children is the lack of care from caregivers towards their children (Talekar et al, 2005), which can be seen as neglect (Chaves et al, 2003). However, not much is known regarding the relationship between dental decay and neglect, which is the aim of this research.

Methods 5-year-old children in a small city (Pacoti, Ceará, Brazil) with a public and well organised dental care assistance program were evaluated for dental problems, utilising the dmft index (number of decayed, missing, or filled teeth in a person), and signs of neglect (general hygiene: dental, hair, fingernail hygiene), through a visual inspection by the same investigator.

Results All 5-year-old children from the municipality were included. A total of 149 children (with parents and their children's consent), from all socio-economic levels, were examined. Only 34.9\% were caries free, and more than $20 \%$ had five or more cavities. $32.9 \%$ had a $\mathrm{dmft}$ index of 4 or more. $64.5 \%$ had bad or partially bad oral hygiene. There was a strong and significant relationship between bad oral hygiene and dental decay experienced $(\mathrm{dmft})(\mathrm{p}=0.001)$. There was also a relationship between general hygiene and hair and fingernail hygiene $(p<0.005)$.

Conclusion These findings suggest that children that are not properly taken care of (that have neglected their general hygiene) are more prone to have dental decay.

\section{SP6-67 TB TRAINING ON DOTS STRATEGY FOR PRIMARY CARE'S HEALTHCARE WORKERS IN 2010, SAO PAULO STATE, BRAZIL}

doi:10.1136/jech.2011.142976q.38

V Souza-Pinto, ${ }^{*}$ V Galesi. Sao Paulo State's Secretary of Health, Tuberculosis Division, Sao Paulo, Sao Paulo, Brazil

Introduction Since establishment of DOTS Strategy in 1998, the Sao Paulo State TB Control Program has always been emphasising the need to sensitise and train healthcare workers (HCWs) in 